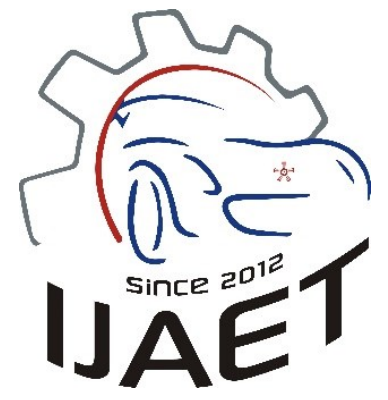

e-ISSN: 2146 - 9067

International Journal of Automotive

Engineering and Technologies

journal homepage:

https://dergipark.org.tr/en/pub/ijaet

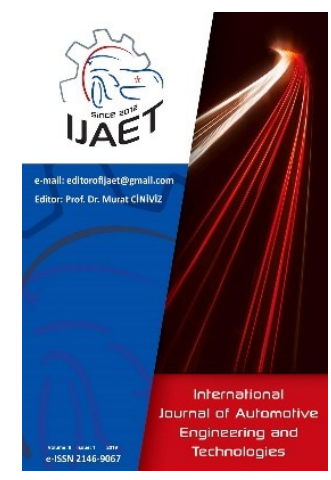

Original Research Article

\title{
Fatigue behavior for design optimization of parking brake bracket connections
}

\author{
Serdar Dalfidan ${ }^{1}$, Haluk Erol ${ }^{2,}$ * \\ 1, 2 * Istanbul Technical University, Mechanical Engineering Faculty, Gümüşsuyu, 34439, Istanbul, Turkey.
}

\author{
ARTICLE INFO \\ $10000-0002-5805-7371$ \\ 2 0000-0003-4230-5921 \\ * Corresponding author \\ erolha@itu.edu.tr \\ Received: Jan 17, 2020 \\ Accepted: Sep 25, 2020 \\ Published by Editorial Board \\ Members of IJAET \\ (C) This article is distributed by \\ Turk Journal Park System under \\ the CC 4.0 terms and conditions.
}

\begin{abstract}
The creation and implementation of brackets have become highly significant in the quality and safety of manufacturing parking brake cables. Moreover, the ability to establish fatigue loads and their effects on the brackets in the vehicle route is now highly important in the design and development process. It is thus crucial to develop an accurate numerical model using computer aided engineering to establish patterns of fatigue during bracket manufacturing. The purpose of this research is to identify the importance of computer aided engineering in bracket optimization, for example in parking brake cables. The key objective of the research is to establish the level of strain exerted on a bracket during oscillation (fatigue) resulting from suspension movement of vehicles. Furthermore, it intends to lower the vehicle's weight and cost by replacing metal brackets with plastic ones.
\end{abstract}

Keywords: Parking brake bracket, Fatigue, Design optimization.

\section{Introduction}

A parking brake cable is a pull cable that is attached on one side to primary cable of hand lever via equalizer, and is attached on the other side to a drum or disc, which differs between models. It is located in the wheels. These cables only transfer traction force and block rotation between certain parts of the drum or disc by pulling on brake spring to create a link between hand brake levers and wheels in vehicles. The route and assembling of this brake cable in the vehicle frame is made up of supporting parts like brackets in different designs, sizes and materials. During vehicle movements, harmonic suspension movements cause loads to fall in different directions and weigh on brackets, however this depends on the condition of the road, i.e. whether they are hollow or bumpy, for example. The loads occurring in different direction and changing cause fatigue on the brackets, ultimately leading to bracket fractures and cracking. These fractures and cracks cause a decline in performance and loss of cable function. For the most part, this is a risk to vehicle safety and can also generate complaints from customers. The examined parking brake system demonstrated in figure 1.

Since it cannot be predicted how fasteners will act during suspension movement, this results in problems and loss of time in the design stages of these elements. The production of each element following the design stages and subjection to physical fatigue tests a form of trial and error, which generates losses of time and money. To ensure that low-cost and high-quality is 
implemented in the short term in the direction of the design process, it is crucial to capture confidence and achieve competitive advantage in the automotive sector. Furthermore, such factors mean it is crucial for the automotive industry to focus on numerical techniques and computer aided engineering to manage such issues. The sub-elements of parking brake cables can be divided into two types according to their functionality contribution, namely direct or indirect elements. Direct functional elements comprise of cable, conduit, cable end fitting and conduit end fitting, whereas indirect functional elements comprise metallic or plastic brackets or fasteners and protector tubes.

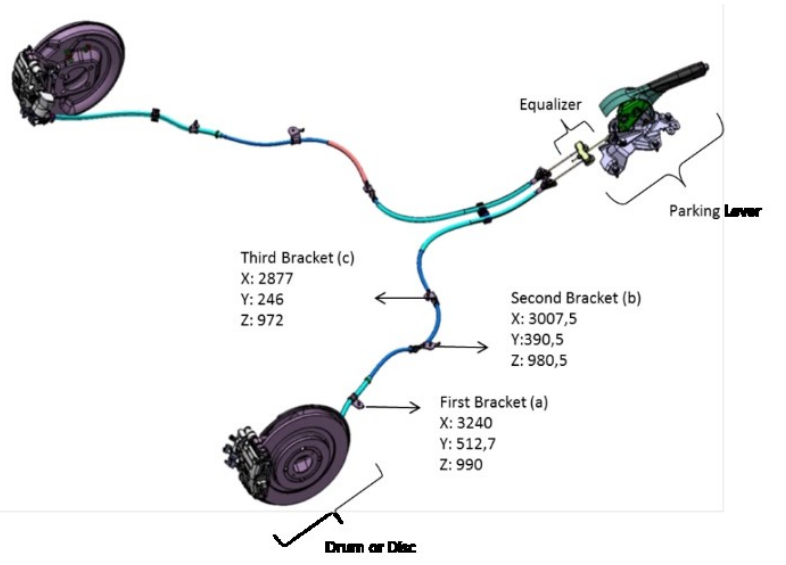

Figure 1: Parking brake system

All parking brake cables possess unique special routes within the vehicle frame which is determined by the vehicle model. Fasteners called brackets are thus commonly used to assign a route and assembly cable within the vehicle frame. The material for the brackets can be selected in accordance with safety level. Plastic brackets should be used in safe route areas and to withhold negligible loads, whilst metallic brackets must only be used in fatigued areas and in places where loads affect the suspension movement. Damage to these connecting elements that are formed and fixed and moving points inevitably causes malfunction resulting from the route's deterioration. Thus, the metal fasteners that are attached to fatigued area, are fundamental to driver safety.

A few studies have been reported on fatigue behavior of parking brake bracket connections. Erol et al. [1] developed a manual transmission gearshift cable and its components in order to control transmitted and propagated noise and vibration to the passenger's compartment and to improve the shift quality of the manual transmission. Kelly and Kent [2] developed a gearshift quality dynamic model using MATLAB. The model takes each of the degrees of freedom and solves for the acceleration of each component either axially or rotationally. Leib et al. [3] investigate the nonlinear dynamic behavior of a gearshift cable and more specifically of the associated tizzing phenomenon. In many studies, cables and brackets have been successfully modeled numerically and parametric analyzes have been carried out to improve their designs [4-6].

The key objective of the research is to establish the level of stress and strain exerted on a bracket during oscillation (fatigue) resulting from suspension movement of vehicles. Furthermore, it intends to lower the vehicle's weight and cost by replacing metal brackets with plastic ones for a $\mathrm{C}$ class vehicle.

\section{Numerical Method}

In the present research, a $\mathrm{C}$ class vehicle model is set to begin a $3 \mathrm{D}$ creation of a parking brake cable. The "critical coordinate" of the brake cable is provided by the based vehicle model and serves as an adequate output for creating the CAD model of the parking brake cable.

This model only intends to include the conduit of brake cable without including other components like inner cable, cable end fittings and conduit end fittings in the 3D model. This is because conduit has a direct impact on brackets in the fatigue test. The 3D model demonstrates a cross section of cable as cylindrical. Furthermore, the spline of route forms part of the vehicle model. The cross section of the cable can be used for the inner liner, single metallic conduit and outer cover which has an outer diameter of $9 \mathrm{~mm}$ and inner diameter measuring $4.4 \mathrm{~mm}$.

The fatigue model also considers other significant elements such as metallic connection elements (see figure 2). The first and third metallic brackets which form part of the parking brake system are $1.5 \mathrm{~mm}$ thick, whilst the second is $2 \mathrm{~mm}$ thick. The first connecting element is directly attached to the suspensions and moves alongside suspension. The second connection element is attached to the frame next to the suspension, where the fatigue loads are 
exerted. The third connecting element is attached to both the vehicle frame and the route of the supporting cable.

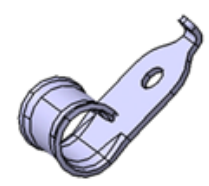

(a)

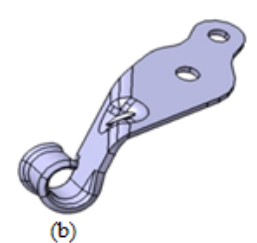

igure 2: CAD model of connecting elements; (a) First bracket, (b) Second bracket, (c) Third bracket

The mid-surface shell technique can be used to model the finite elements relating to metal brackets. Developing the mid-surface rather than a three-dimensional model of all elements is beneficial in that is make sure that finite elements are separated accurately and homogeneously.

These 3D models will be utilized in order to construct the finite element model must first be entered into the finite element program. The element of conduit, is inputted as a 3DDeformably model and brackets are inputted as a middle surface shell model on finite element model. The material used to make each element is identified and assigned to each element in the finite element model. The finite element method is selected because it is "dynamic implicit" for a time-depended model. Movement directions and extent of movement are used in the model with boundary conditions and finite element modelling is concluded by categorizing elements into finite elements using the mesh technique. In accordance with specified boundary conditions and the connections between elements, the finite element model is solved and output data is gathered from critical bracket (otherwise known as the second bracket).

Boundary conditions that are inserted into the finite element model are calculated according to fatigue data in the parking brake cable and will vary in accordance with the selected vehicle model (as seen in figure 3). The displacement and rotation data is a key boundary condition of the system.

When developing the finite element model, the top point of the 3-D fatigue model relating to parking brake cable must be regarded as the reference. The fatigue motion established using the 3D fatigue model occurs around the axis with the starting point $\mathrm{x}=3070.88, \mathrm{y}=692.39$, $\mathrm{z}=1020.12$ and end point $\mathrm{x}=3070.88, \mathrm{y}=$ -
692.39, $\mathrm{z}=1020.12$. There is $28^{\circ}$ movement around this axis with a $60.13 \mathrm{~mm}$ route taken by this axis group. Inputs to the finite element system are thus essential; $U_{\mathrm{rx}}: 0.49 \mathrm{rad}$ and $\mathrm{U}_{\mathrm{z}}$ : $60.13 \mathrm{~mm}$. The boundary conditions attached to fixing points are defined so the connecting elements will be positioned in the holes secured with screws. Other fatigue data applied throughout the analysis will be the frequency of the fatigue motion. This frequency is 2.5 cycles per second.

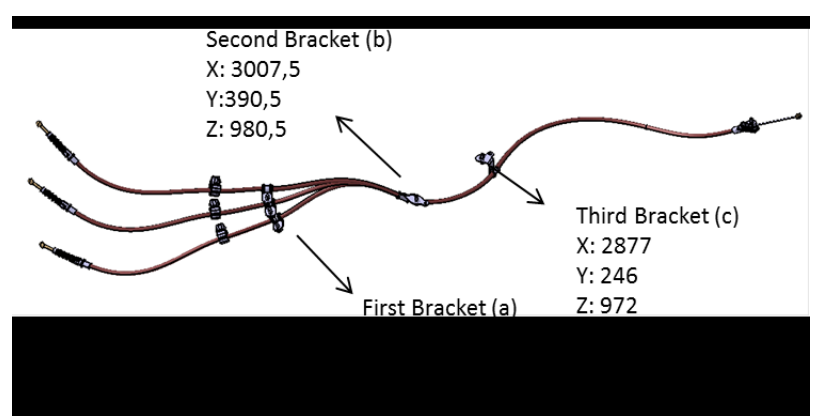

Figure 3: CAD model of parking brake cable

The finite element model is resolved in the final stage of the fatigue model's solution process. This was achieved by observing and measuring the inputs specified in the boundary conditions. As shown in figure 4, the system is applied for meshing method and implemented input boundary conditions to pertain to strain on the second bracket, (also known as the critical element in figure 3), were also worked out.

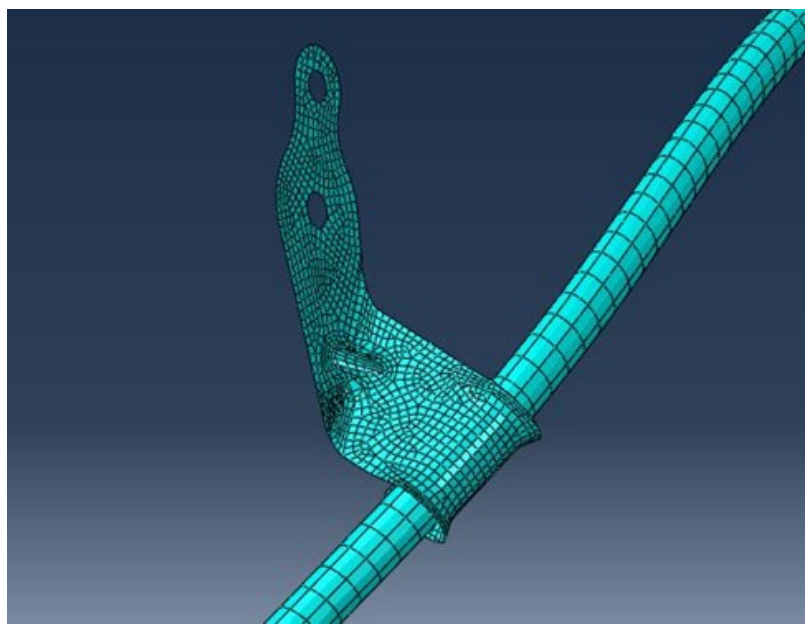

Figure 4: CAE simulation meshing method

These strain values are calculated by measuring the bracket area which receives the strain. This is known as the experimental validation process and is presented in figure 5. This area is selected to implement experimental method by Strain Gauge during oscillation test.

Figure 6 presents minimum and maximum 
principal strain graphs by time. As seen from graphs that minimum principal strain value is around $0.00008 \mathrm{~mm} / \mathrm{mm}$ and maximum principal strain value is around 0.000012 $\mathrm{mm} / \mathrm{mm}$. The numerical model has been verified with these data.

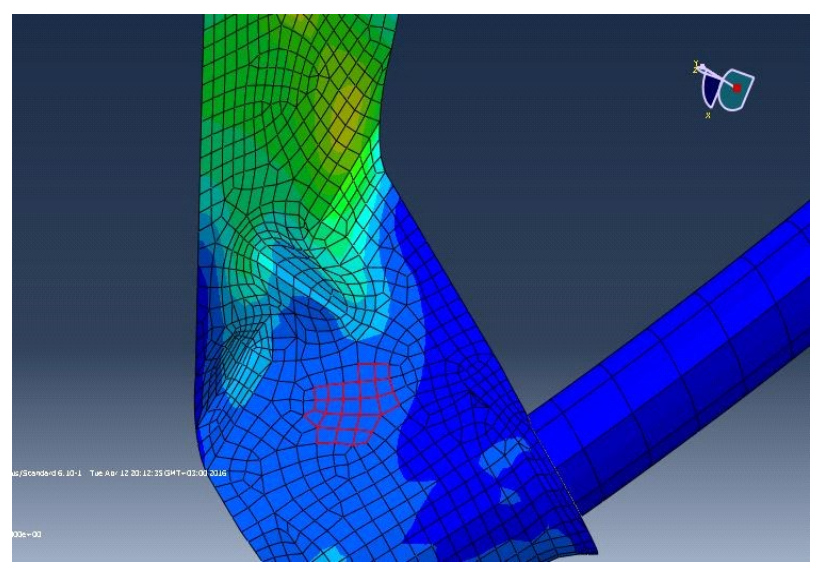

Figure 5: The area on second bracket applied strain gauge

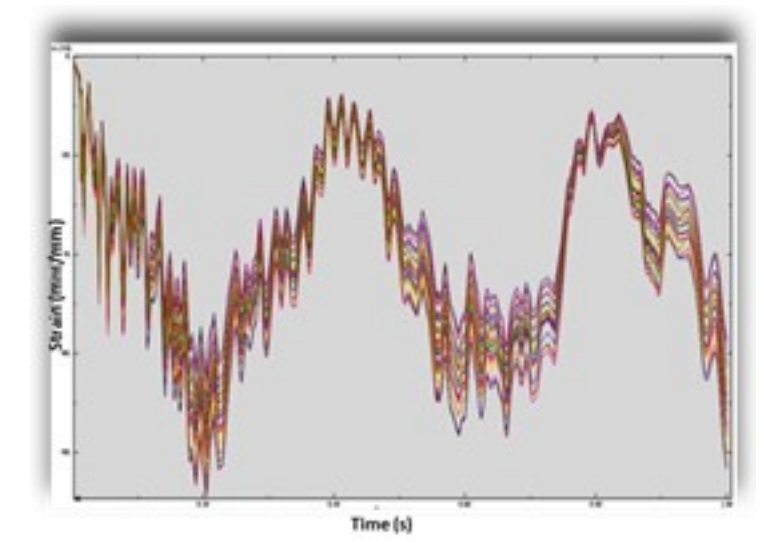

(a)



(b)

Figure 6: (a) Minimum principal strain; (b) Maximum principal strain

Most of the damage that happens to the components in the real world is a result of the load cycle. The load is dynamic can be applied in cycles. This leads to fatigue on the component, which gradually wears down due to this fatigue mechanism.

NCode software program is used to assess the life and damage of a parking brake cable by making numerical calculations. It is used in the present study. The aim of NCode fatigue analysis is to establish the extent of the damage in a 1-unit system and to determine the continuity of a system in accordance with 1-unit motion. It is also used to work out the total damage in the desired quantity.

If the strain is exerted in fatigue model of parking brake system for 2.5 cycles in one second, then the damage factor must be assessed for a one second motion.

Figure 7 presents the NCode fatigue analysis program and the created flow chart, in which the material properties must be established firstly by applying parameters of the near-by feature in the NCode software program based on the features of the metal brackets.

After creating the NCode model, the total amount of damage on the front and rear fastener surface was subsequently measured for one second of movement. The target cycle amount outlined in the base vehicle model regulations is 1000000 cycles.

This demonstrates that total damage following 1000000 cycles was 0.1125 , which falls below 1 , and can thus be deemed safe. The following figure 8 shows the overall damage.

The other crucial design and development process is the optimization of structures which possess better functionality.

There are two key optimization types: material optimization and size (dimensional) optimization. Material optimization can be applied to alter the mechanical properties of the material or of the material microstructure. Different material optimization levels can be used in accordance with different variables. Size optimization may be applied to alter the cross section or thickness of structure as a means of enhancing the structure's durability or increasing the material used to lower the cost and weight.

Plastic bracket is designed as two separated parts which is assembled by nails to each other. Figure 9 below presents the design of the plastic bracket:

Throughout the process of optimizing the metallic bracket, three applicable plastic bracket optimization is selected due to the change of material properties and thickness quality (see table 1). 


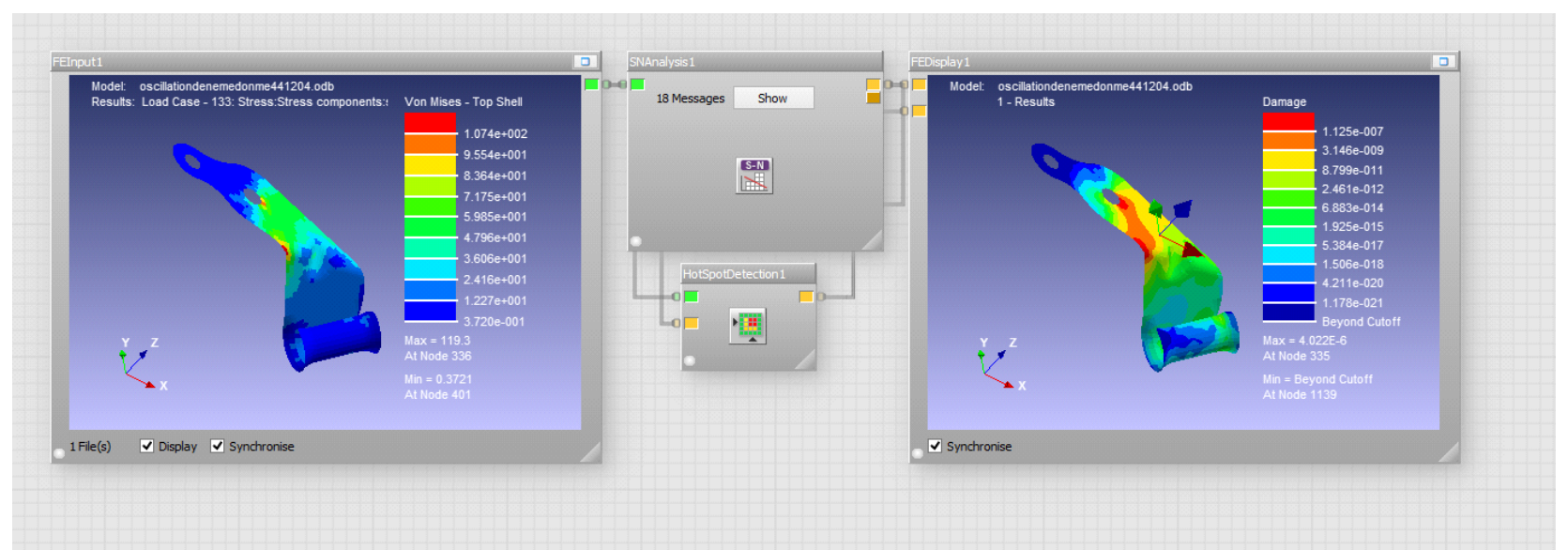

Figure 7: Flow chart of NCode



(a)

(b)

Figure 8: Total damage status of connection bracket after NCode analysis (a) Rear; (b) Front

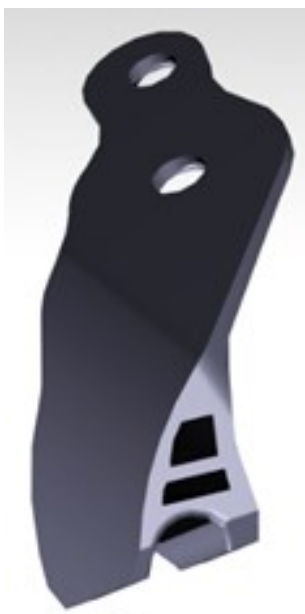

(a)

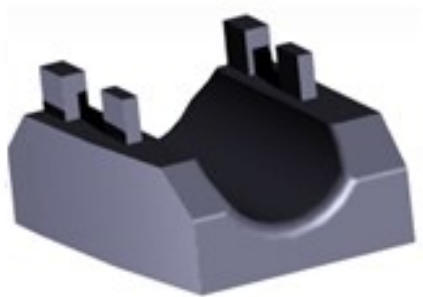

(b)

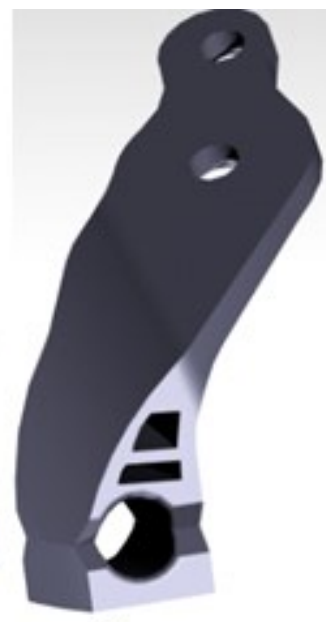

(c)

Figure 9: Plastic bracket design; (a) Upper bracket, (b) Lower bracket, (c) Assembly bracket

Table 1: Applicable plastic brackets

\begin{tabular}{ccc}
\hline Case \# & Thickness [mm] & Material \\
\hline 1 & 4 & PA66 GF50 \\
2 & 4 & PA66 GF30 \\
3 & 2 & PA66 GF50 \\
\hline
\end{tabular}

\section{Experimental Studies}

The first step in any experimental study is to identify and produce material data that can subsequently be entered into the finite element model. Accurate detection of such inputs and effective use of these inputs in the model enhances the accuracy of findings produced by the finite element model.

The second step in the research study following numerical analysis is to use the finite element model of materialized specimens to create a physical model in order to verify the accuracy of 
the data produce. Its primary objective was to determine the loads exerted on critical bracket using the strain gauge as support through the fatigue (oscillation) test method. It is thus assumed that physical data produced will be very similar to the numerical data acquired using the finite element model.

The conduit, one of the most fundamental components of the parking brake cable system is largely considered throughout the research. Furthermore, the component is made up of three separate layers and possesses a composite structure as shown in figure 10 . This composite structure adds the metal single-stranded cable, (a crucial additive spring feature) to the material properties. As well as this composite structure, the inner cable (which is crucial in allowing the parking brake cable to function) also offers an additive. In the present research study, rather than exploring all the components as separate entities, a single piece was utilized to determine the elasticity modulus.

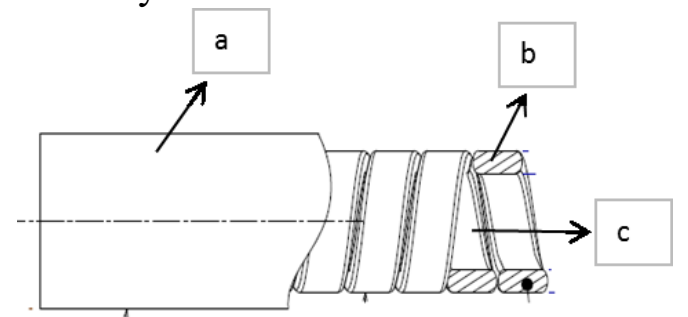

Figure 10: Conduit layers; (a) PBT cover, (b) Metal layer, (c) PTFE inner liner

Firstly, it is crucial to assess the rigidity of the composite structure in order to determine elasticity. This stiffness is a variable applied in measuring the bending behavior of the parking brake cable throughout the setting up of the finite element model. The bending stiffness of the conduit was establishing by applying the three-point bending method demonstrated in figure 11.



Figure 11: Three-point bending method

The three-point bending test apparatus comprises of two simple supports, a load cell and a push rod (which is presented in figure 12).

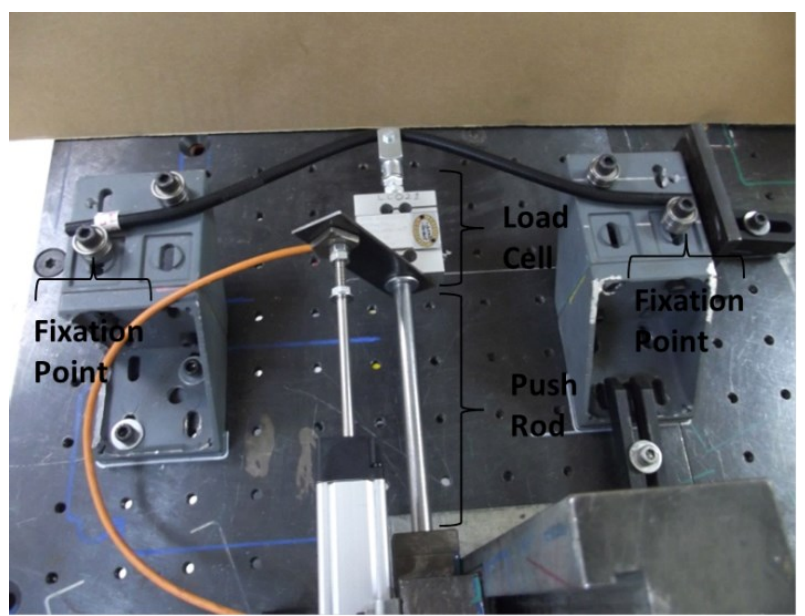

Figure 12: Load-cell and test setup

Five samples were assessed using a three-point bending method in an attempt to determine the average stiffness value. Figure 13 shows a forcedisplacement graph relevant to sample \#1.

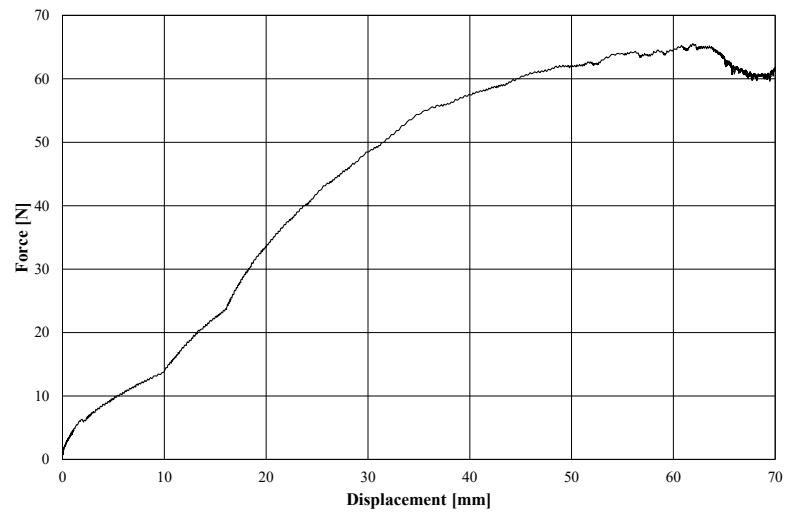

Figure 13: Force-displacement graph of sample \#1

The flexural rigidity of the sample (as seen in figure 14) was worked out to calculate the stiffness data which was to be transferred to the finite element model.

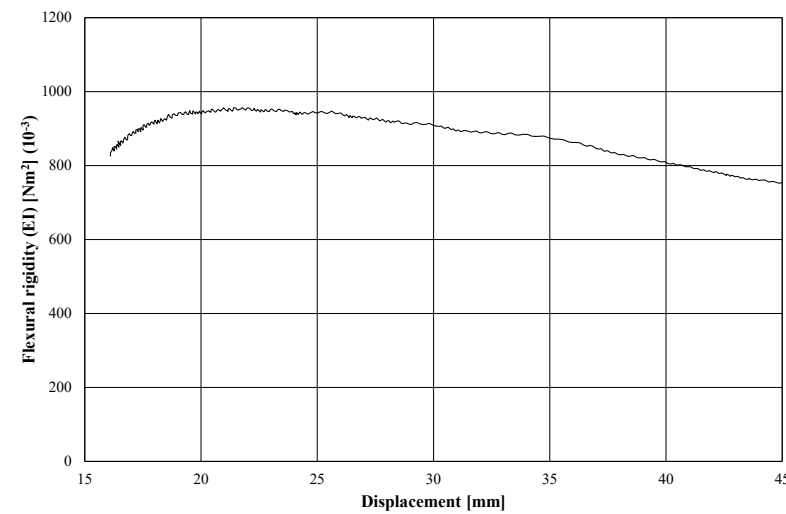

Figure 14: Flexural rigidity according to displacement of sample \#1

As the composite structure applied in the creation of the parking brake system is cylindrical, the structure's moment of inertia can be worked out cylindrically. Therefore, 
elasticity modulus value can be worked out directly using the bending stiffness. In respect of this calculation, a graph presenting the elasticity modulus relevant to sample \#1 can be seen in figure 15 .

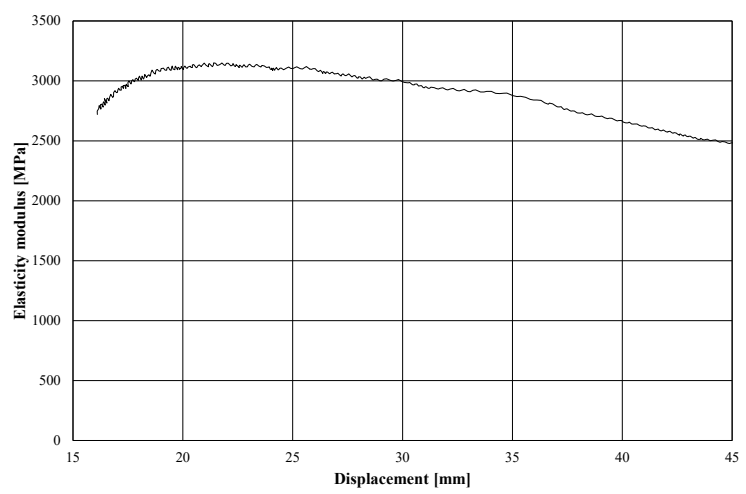

Figure 15: Elasticity modulus graph of sample \#1

In total, five specimens were evaluated in terms of their elasticity modulus. It was subsequently found that the modulus of elasticity and average elasticity were 3015.48 MPa as presented in table 2 below.

Table 2: Average elasticity modulus of samples

\begin{tabular}{cc}
\hline Sample \# & Average Elasticity Modulus (MPa) \\
\hline 1 & 3156.81 \\
2 & 3229.51 \\
3 & 2801.85 \\
4 & 3000.63 \\
5 & 2888.60 \\
Average & $\mathbf{3 0 1 5 . 4 8}$ \\
\hline
\end{tabular}

The second feature conduit is the structural damping factor and is a key additive to fatigue behavior of the system. Hysterical graph method is used to assess the structural damping factor. The hysterical graph method is applied in order to work out the energy loss that happens during a fatigue cycle.

The three-point bending method is applied to work out the conduit elasticity modulus was applied continuously to establish the conduit's actions when bouncing back to a particular point. As previously stated, the return hysteresis curve of the loop cable is worked out according to force-displacement (see figure 16).

The values of $\eta=0.0573$ and $\zeta=0.0267$ are worked out respectively. The structural damping factor " $\zeta$ " must be applied to the finite element model in this case as an input to the conduit material parameters.

The final characteristic of conduit is a collaborated density of composite structure. A density meter is applied to work out the average conduit density. The average density value that will be entered into the finite element model is 5.43 tone $/ \mathbf{m m}^{2}$ for conduit.

The metal brackets represent a further structure that is applied in the finite element model. The brackets comprise of DC04 sheet metal, which is highly appropriate for cold forming. Table 3 shows the DC04 sheet metal material parameters, which must be entered into the finite element model in accordance with the EN 10130 norm.

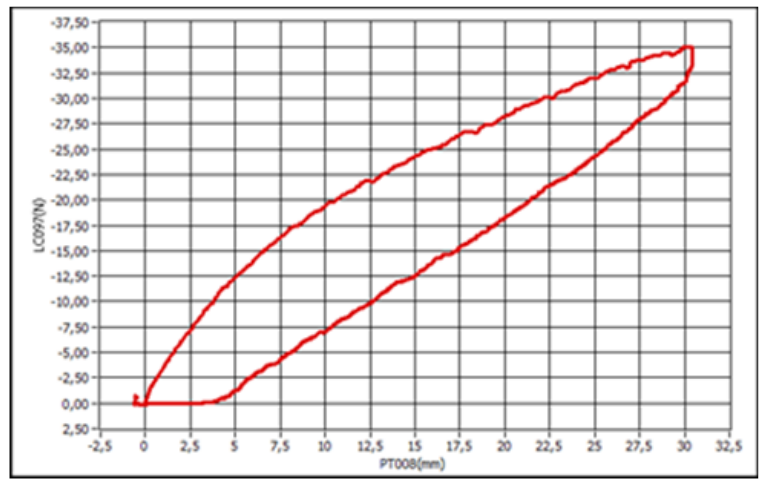

Figure 16: Hysterical graph of conduit

Table 3: Material parameters of metallic bracket

\begin{tabular}{ccc}
\hline$\delta$ (Density) & $\begin{array}{c}\text { E (Elasticity } \\
\text { Modulus) }\end{array}$ & $\begin{array}{c}v \text { (Poisson } \\
\text { Ratio) }\end{array}$ \\
\hline 7891 & $210 \mathrm{GPa}$ & 0,3 \\
\hline
\end{tabular}

Fatigue can be defined as the wear on a system caused by a certain displacement and frequency oscillation. Fatigue test equipment can be applied in parking brake cables to represent the oscillating motion that happens are the suspension move when the vehicle is in motion. The key objective of using fatigue testing devices is to establish the level of damage to the brake cable that results from oscillating motion. To work out the strain on a critical bracket, a strain gauge device is used alongside the finite element model of parking brake cable. The strain gauge rosettes are combinations that enable the strain gauge pieces to be located in close proximity to each other in two places in order to assess the average strain that is exerted when the test piece is set in different directions. In this case, the type and orientation of the rosette must be in line with the information provided regarding the tension meter to gain data from the fatigue test regarding the damage to the critical metal bracket element. A "planar, $45^{\circ}$ - rectangular" type (presented in figure 14) badge was attached to the metal connection element and the strain measurement was assessed in three directions. Tables 4 and 5 present the rosette properties and sizes. 
Table 4: Dimensions of strain gauge rosette

\begin{tabular}{cccccc}
\hline $\begin{array}{c}\text { Gage } \\
\text { Length }\end{array}$ & Total Length & $\begin{array}{c}\text { Wire } \\
\text { Thickness }\end{array}$ & $\begin{array}{c}\text { Total } \\
\text { Thickness }\end{array}$ & $\begin{array}{c}\text { Matrix } \\
\text { Length }\end{array}$ & $\begin{array}{c}\text { Matrix } \\
\text { Thickness }\end{array}$ \\
\hline $0.062 \mathrm{inch}$ & $0.185 \mathrm{inch}$ & $0.052 \mathrm{inch}$ & $0.260 \mathrm{inch}$ & $0.247 \mathrm{inch}$ & $0.410 \mathrm{inch}$ \\
$1.520 \mathrm{~mm}$ & $4.700 \mathrm{~mm}$ & $1.270 \mathrm{~mm}$ & $6.600 \mathrm{~mm}$ & $7.040 \mathrm{~mm}$ & $10.410 \mathrm{~mm}$ \\
\hline
\end{tabular}

Table 5: Properties of strain gauge rosette

\begin{tabular}{ccccc}
\hline & $\begin{array}{c}\text { Gage Factor @ 24 } \\
\text { \% }\end{array}$ & $\begin{array}{c}\text { Transverse } \\
\text { Sensitivity }\end{array}$ & $\begin{array}{c}\text { GAGE Resistance } \\
\text { (Ohm) }\end{array}$ & $\begin{array}{c}\text { Gage Factor @ } \\
\mathbf{1 0 0}^{\circ} \%\end{array}$ \\
\hline Gage 1 & $2.110 \pm 0.5 \%$ & $+1.7 \pm 0.2 \%$ & & \\
Gage 2 & $2.175 \pm 0.5 \%$ & $+0.8 \pm 0.2 \%$ & $350 \pm 0.6 \%$ & $+1.2 \pm 0.2$ \\
Gage 3 & $2.110 \pm 0.5 \%$ & $+1.7 \pm 0.2 \%$ & \\
Nominal & $2.140 \pm 2.0 \%$ & & \\
Lote Number & A $86 \mathrm{BD} 826$ & & \\
\hline
\end{tabular}

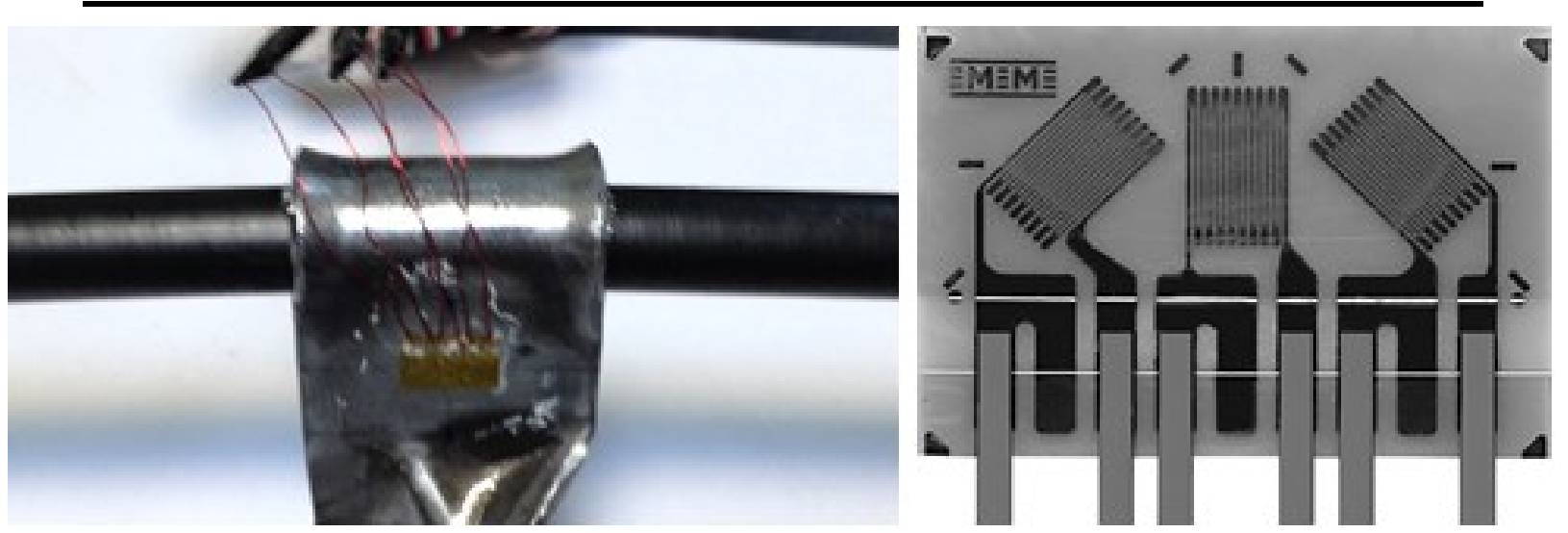

Figure 17: (a) Strain gauge point on critical bracket; (b) Channel on rectangular rosette

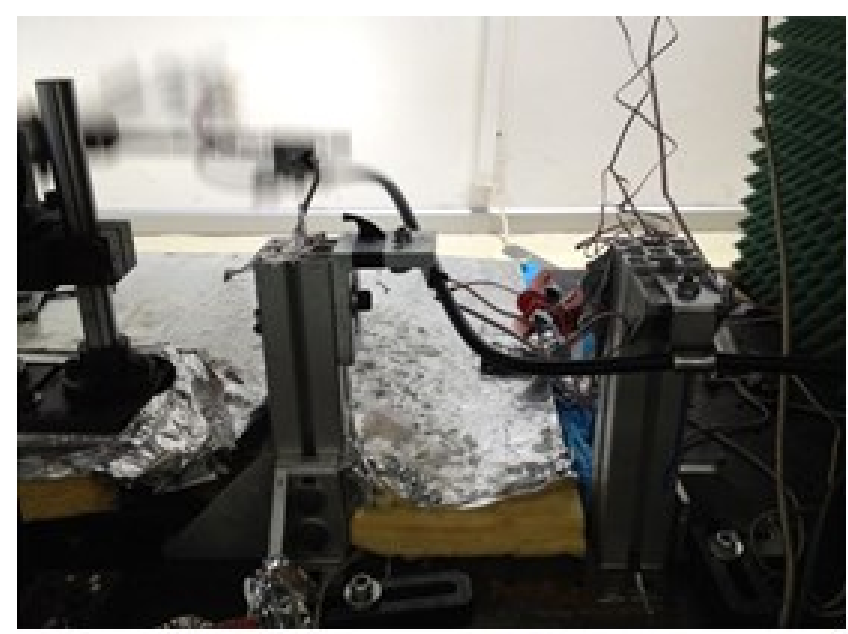

(a)

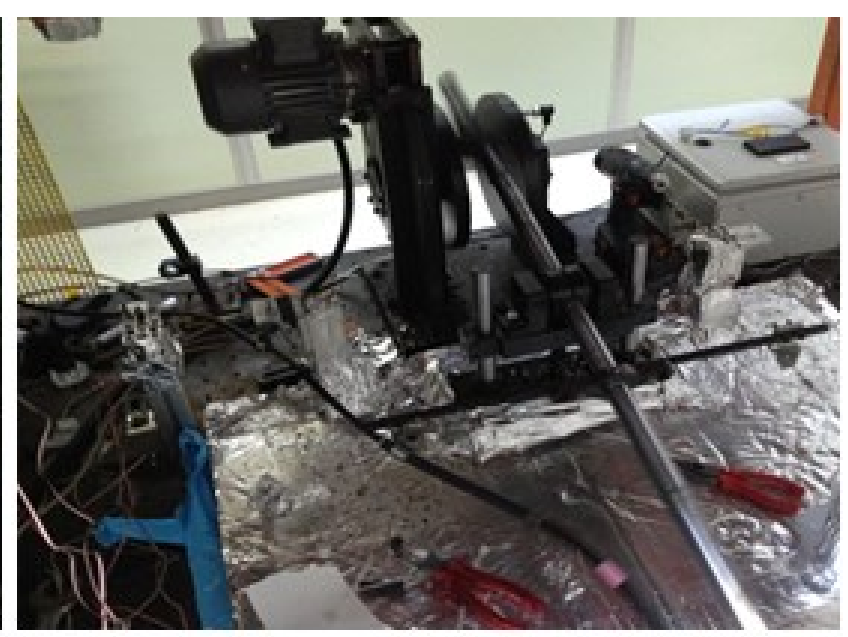

(b)

Figure 18: (a) Route of parking brake cable in oscillation test bench; (b) Fatigue movement and data transfer on bench

The selected strain gauge position can be seen in figure $17(\mathrm{a})$, and is the most appropriate connection element with the most suitable flatness based on the rosette size. Strain values for pressure on the connection element were recorded in three directions and were noted down in micrometers (see figure 17).

After applying the strain gauge rosette, the corresponding parking brake cable must be joined to the oscillation test bench in its own direction (demonstrated in figure 18(a)). After setting the required program settings and device connections, the test can begin and data transmission through the strain gauge can be conducted, as seen in figure 18(b). The tension meter must be reset at zero when the oscillation bench is located in top position (the point of minimum tension), and the top point is deemed to be the reference point.

The data taken recorded for the three directions using three different channels of the strain gauge were presented in the following chart as seen on 
figure 19.

Strain values with indefinite indications were rotated by applying prime principal equations taken from the Mohr Circle as a means of reclaiming values (see figure 20 ).

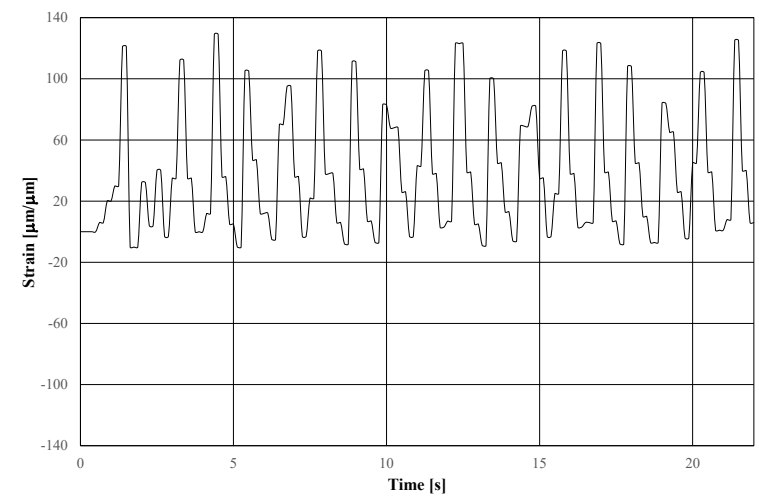

(a)

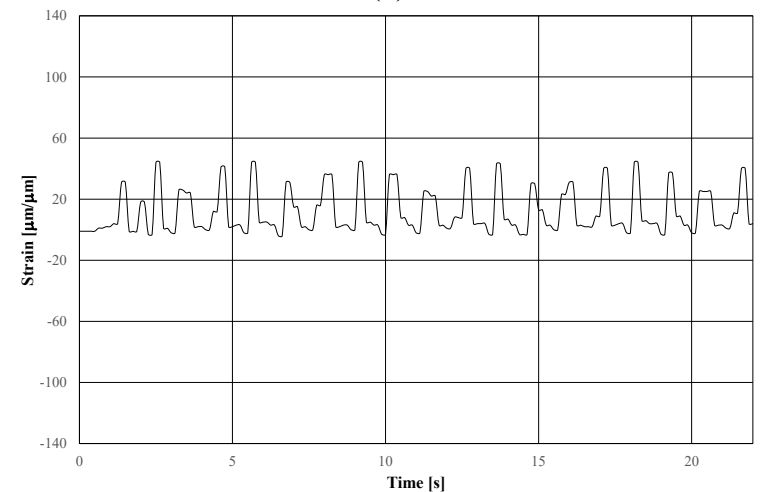

(b)

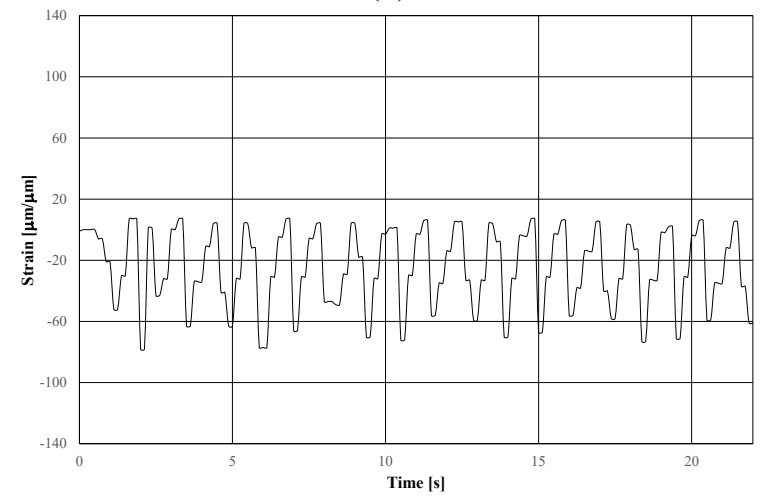

(c)

Figure 19: The strain data from three different directions; (a) E1, (b) E2, (c) E3

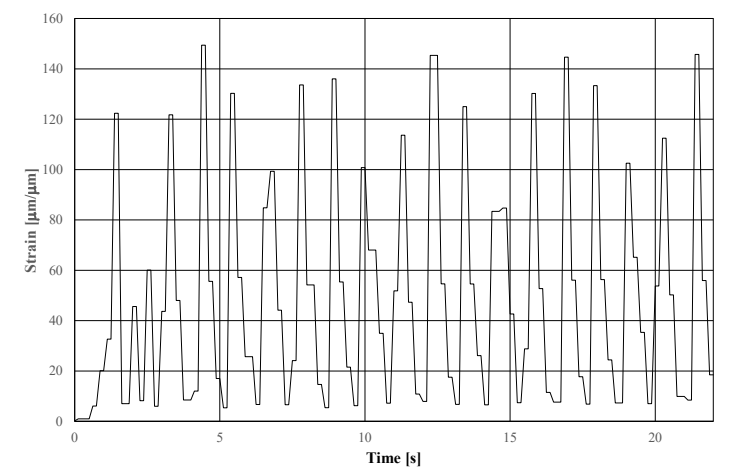

(a)

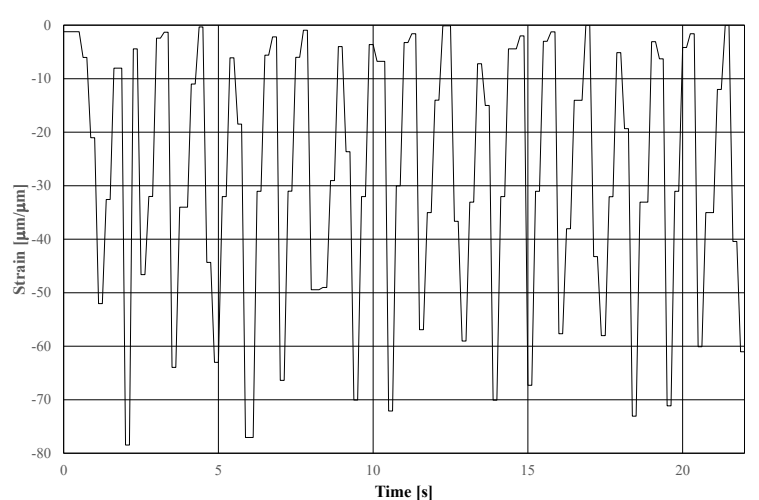

(b)

Figure 20: (a) Maximum principal strain; (b) Minimum principal strain

\section{Results and discussion}

The purpose of the present research was to explore the fatigue behaviors of the connection elements used in the parking brake cable system and to enhance these elements using computer aided engineering. When the numerical and the experimental results are evaluated together, it is seen that the numerical model gives realistic results. This should presumably mean that the final result, which will be acquired using trial and error methods in the design or development process, will be achieved in a quick and inexpensive manner.

Furthermore, it intends to lower the vehicle's weight and cost by replacing metal brackets with plastic ones. Such brackets are crucial supporting components in the hand brake structure and play a vital role in its function. By creating brackets that can perform similar functions at a similar level but with less cost, the plastic bracket can be optimized using various materials and compatible design.

Optimization research indicates that plastic bracket is easier to apply and possess a more appropriate design and suitable material selection than the metal apparatus.

The figure 21 shows data pertaining to the comparison cases versus maximum strain values. It shows that optimizations 2 and 3 have lower strain values the metal apparatus and have similar strain values within themselves. Optimization 1 shows strain values at a much lower level.

Damage at 1000000 cycle life time for 4 cases can be seen in the comparisons made in figure 22.

Another key point to consider in optimization research is comparing the weight of materials 
used for parts. Figure 23 clearly demonstrates that plastic brackets in three cases are lighter and thus have the advantage of reducing weight. The use of a plastic bracket instead of metallic bracket reduces weight by approximately $80.6 \%$.



Figure 21: Maximum strain values

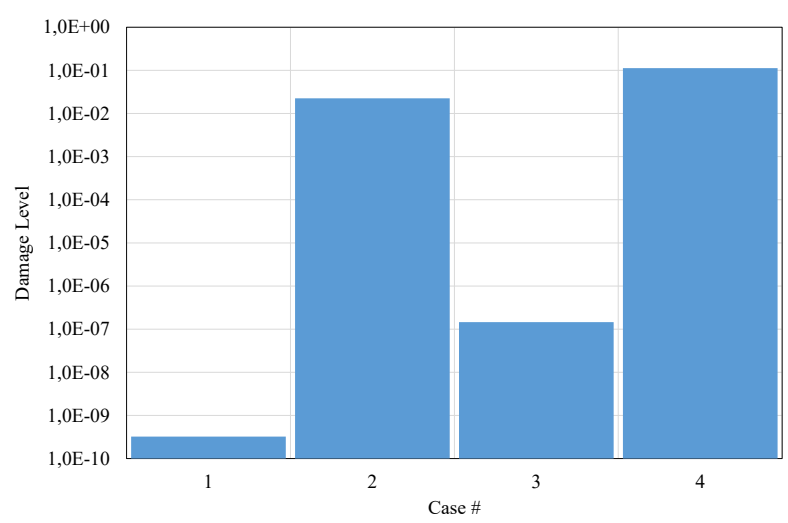

Figure 22: Damage levels



Figure 23: Total weight values

A further requirement in optimization is to lower costs or at least equal the current cost. Figure 24 presents prices comparison for metallic bracket and plastic bracket cases. Plastic case 2 are more affordable $(0.1191 €)$ that the other options.

\section{Conclusions}

The purpose of this research was to identify the importance of computer aided engineering in metallic bracket optimization, for example in parking brake cables. The key objective of the research is to establish the level of strain exerted on a bracket during oscillation (fatigue) resulting from suspension movement of vehicles. Furthermore, it intends to lower the vehicle's weight and cost by replacing metal brackets with plastic ones. Research indicates that Case \#2 is the best choice in terms of combining performance.

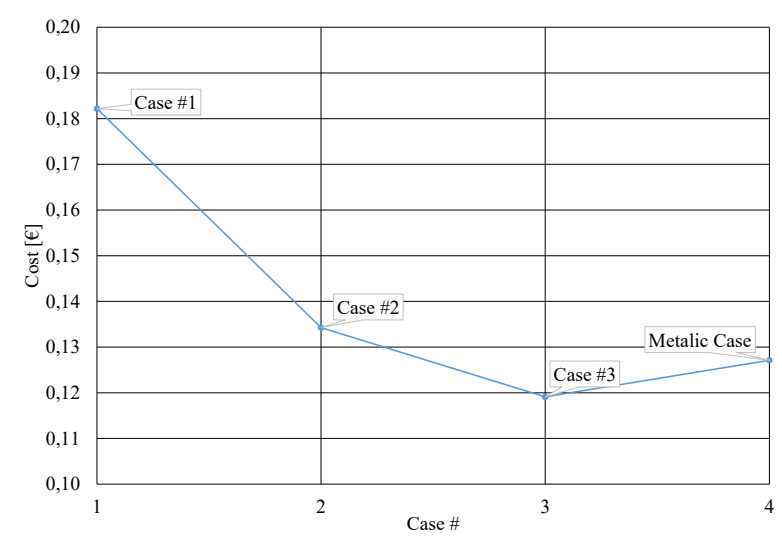

Figure 24: Cost values

\section{References}

1. H. Erol, H. Karabulut and K. Kaynar, "On the dynamics of a gearshift system in manual transmission", Inter-noise 2013.

2. D.K.C. Kent, "Gear shift quality improvement in manual transmissions using dynamic modeling", FISITA World Automotive Congress, 2000.

3. N. Leib, S. Nacivet and F. Thouverez, "Experimental and numerical study of a vibroimpact phenomenon in a gearshift cable", Journal of Sound and Vibration, 329, 289-301, 2010.

4. S. R. Ghoreishi, T. Messager, P. Cartraud and P. Davies, "Validity and limitations of linear analytical models for steel wire strands under axial loading, using a 3D FE model", International Journal of Mechanical Sciences, 49(11), 1251-1261, 2007.

5. M. L. Tang, Y. G. Du, R. L. Shen and K. Yan, "Study of the bending stiffness and cable characteristics of tension components", Applied Mechanics and Materials, 188, 17-24, 2012.

6. X. Ma and J. W. Zhang, "Finite element analysis and the topology optimization of a bracket-cable", Applied Mechanics and Materials, 44-47, 1325-1329, 2010. 